\title{
BMJ Open Multicentre, prospective registry study of amyotrophic lateral sclerosis in mainland China (CHALSR): study protocol
}

\author{
$\mathrm{Ji} \mathrm{He},{ }^{1}$ Jia yu Fu, ${ }^{1}$ Lu Chen, ${ }^{1}$ Jing He,${ }^{2}$ Jingxia Dang, ${ }^{3}$ Zhangyu Zou, ${ }^{4}$ Sha Ma,${ }^{5}$ \\ Nan $\mathrm{Li}^{6}{ }^{6}$ Dongsheng Fan (D) ${ }^{1}$
}

To cite: He J, Fu Jyu, Chen L, et al. Multicentre, prospective registry study of amyotrophic lateral sclerosis in mainland China (CHALSR): study protocol. BMJ Open 2020;10:e042603. doi:10.1136/ bmjopen-2020-042603

- Prepublication history for this paper is available online. To view these files, please visit the journal online (http://dx.doi org/10.1136/bmjopen-2020042603).

Received 09 July 2020 Revised 08 0ctober 2020 Accepted 16 0ctober 2020

\section{Check for updates}

(C) Author(s) (or their employer(s)) 2020. Re-use permitted under CC BY-NC. No commercial re-use. See rights and permissions. Published by BMJ.

${ }^{1}$ Neurology, Peking University Third Hospital, Beijing, China ${ }^{2}$ Neurology, Beijing Hospital, Beijing, Beijing, China

${ }^{3}$ Neurology, First Affiliated Hospital of Xi'an Jiaotong University, Xi'an, Shaanxi, China ${ }^{4}$ Neurology, Fujian Medical University Union Hospital, Xiamen, Fujian, China ${ }^{5}$ Neurology, The First People's Hospital of Yunnan Province, Kunming, Yunnan, China

${ }^{6}$ Research Center of Clinical Epidemiology, Peking University Third Hospital, Beijing, China

Correspondence to

Dongsheng Fan;

dsfan2010@sohu.com

\section{ABSTRACT}

Introduction Amyotrophic lateral sclerosis (ALS) is a representative rare disease characterised by progressive, fatal motor neuron degeneration. Due to the unknown aetiology and variability of the phenotypes, there are no accurate reports concerning the epidemiology or clinical characteristics of the disease. The low prevalence, as previously reported, makes it difficult to carry out studies with large samples. The aim of this study was to explore the natural history and clinical features of ALS in mainland China through a multicentre, prospective cohort study. The findings will both offer a better understanding of ALS and also support the development of a model to study other rare diseases.

Methods and analysis Patients from 88 representative hospitals in different parts of mainland China will be recruited through a specially designed online data system (http://www.chalsr.net). We aim to recruit 4752 ALS patients over a 3-year period. Baseline data will be recorded, and follow-up data will be collected every 3 months. The primary outcome is effective survival. Overall survival and indices of disease progression will be measured as the secondary outcomes.

Ethics and dissemination Ethical approval has been obtained from the ethics committee of Peking University Third Hospital (M2019388). Informed written consent will be obtained from each participant. Dissemination of the study protocol and data will take place primarily through a specially designed online data system (http://www.chalsr. net). The collective results of the study will be published in peer-reviewed journals and shared in scientific presentations.

Trial registration number NCT04328675

\section{INTRODUCTION}

In the past two decades, increasing attention has been paid to rare diseases, which impose enormous burdens on both patients and society worldwide. Amyotrophic lateral sclerosis (ALS) is one of the most representative rare diseases, characterised by progressive, fatal motor neuron degeneration. It is the typical type of motor neuron disease. ${ }^{12}$ The onset of the disease in middle age and
Strengths and limitations of this study

- This study will be the largest multicentre, prospective study of amyotrophic lateral sclerosis in mainland China.

- Data from hospitals of different sizes located in different geographical regions of China will be included.

- The design includes a follow-up evaluation every 3 months throughout the study.

- A specially designed online data system will be used to unify the study and develop a model to study other rare diseases.

- Due to the uneven distribution of medical resources in China, our study may have information biases.

the clinical manifestation of systemic paralysis lead to a substantial economic burden of up to US\$30934 (£23 165) per annum for each patient. $^{3}$ The worldwide all-age prevalence and incidence of motor neuron disease are 4.5 per 100000 people and 0.78 per 100000 person-years, respectively. ${ }^{4}$ However, recent epidemiological studies of ALS show great regional and temporal variations, with higher rates in West Europe than in South Asia and a significant increase in the incidence by 0.00013 per year. ${ }^{5}$ The low prevalence and incidence increase the difficulty of ALS research. Population-based registers or multicentre cohorts assure the large sample size and intact follow-ups, which greatly impulse our understanding of the disease, such as the European ALS Consortium (EURALS), the European Multidisciplinary ALS Network Identification to Cure Motor Neurone Degeneration (Euro-MOTOR) and the National ALS Registry. ${ }^{6-9}$ Those studies substantially revealed epidemiology and natural features of ALS. ${ }^{10}$ However, such large research is rare in Asian area. As the population size is of more than 1.3 billion in China, it would be 
a productive exploration for implementing large registry studies. $^{11}$

Another major challenge that creates a dilemma for ALS research is the high heterogeneity of the disease. According to previous research, the natural history and clinical features of ALS vary based on different phenotypes, genotypes, ethnicities, environmental backgrounds and lifestyles. ${ }^{12-15}$ The aetiology and prognostic factors are often unpredictable, which limits targeted treatment and ultimately causes high mortality. ${ }^{16}$ This situation creates an urgent need for multicentre studies with widespread geographical reach and regular follow-up to evaluate different aspects of the disease, including its regional and temporal distribution, its progression and the factors affecting the outcomes. ${ }^{1718}$

As a representative rare disease, ALS faces a common weakness of rare diseases in that it is distributed sparsely among sporadic patients and qualified regional institutions. Thus, an internet-based mobile terminal that can share registered cases from different regions will be a perfect tool to solve the uneven distribution of patients and medical resources. ${ }^{19}$ In order to improve the level of diagnosis and treatment, it is important to have a standard operating procedure for management formulated by a recognised authoritative institution that has sufficient experience with the specific disease. ${ }^{20} 21$ By combining an internet-based platform with unified professional management of ALS, we hope to establish a new standard model for the study of rare diseases.

Thus, the registry study of ALS in mainland China (CHALSR), the first multicentre, prospective registry study of ALS, is expected to provide an accurate description of ALS in China with a considerable quantity of patients. We hope the study will establish a stable cooperation platform and guide the management of future research on ALS.

\section{OBJECTIVES}

The aim of our study was to explore the natural history and clinical features of ALS in mainland China through a multicentre, prospective cohort. The specific objectives include the following:

- Investigate the geographical and temporal distribution of ALS patients in mainland China from 2020 to 2023.

- Describe the clinical characteristics and disease progression of ALS.

- Identify the factors that impact the prognosis of ALS.

\section{METHODS AND ANALYSIS \\ Study design}

This is a multicentre, prospective registry study that will be conducted using a unified ALS-specific internet platform. The standard operating procedure for the overall design and implementation of the study was developed by Peking University Third Hospital (PUTH), the largest ALS centre in China. Hospitals that have ALS centres

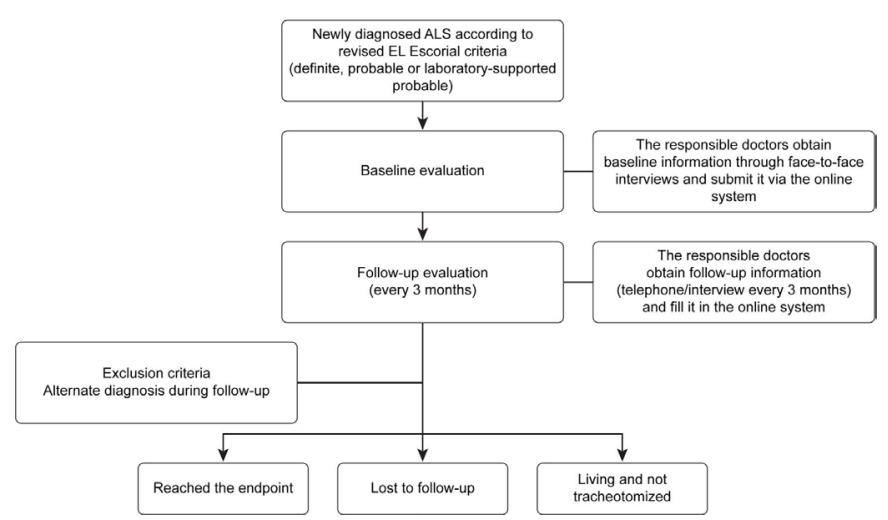

Figure 1 Flow chart of the overall study design and process. ALS, amyotrophic lateral sclerosis.

and are willing to use the platform will be included. Each participating hospital will have one principal investigator who will carry out the study recruitment process and the overall supervision. The collaboration partners from each centre will be trained to use the internet platform to share the data according to a standardised method. In all cases, baseline demographic information and clinical data will be collected during the patient's first visit to the centre and during follow-up evaluations conducted by telephone or in person every 3 months throughout the study. Patients will be recruited from March 2020 to at least March 2023. Figure 1 illustrates the specific procedure for study recruitment and the overall flow of eligible subjects.

\section{Study participants}

Inclusion criteria

- Patients aged 18-80 years.

- Patients diagnosed with definite ALS, probable ALS or laboratory-supported probable ALS by an experienced neurologist according to the revised El Escorial criteria.

- Patients who sign informed consent.

\section{Exclusion criteria}

- Patients who receive alternative diagnoses during follow-up.

- Patients who refuse to sign informed consent.

\section{Study sites and sample sizes}

This study will be performed at centres spread across approximately 20 provinces, four municipalities and four autonomous regions in mainland China (figure 2). At least one tertiary first-class hospital from each of the participating provinces will take part in the study. A total of 88 hospitals will be involved.

According to the pilot study, the median of patients diagnosed with ALS and completing the detailed clinical follow-ups in our participating hospitals is 18 per year. Thus, based on clinical experience, the total number of patients included in this 3-year study is theoretically estimated to reach 4752 . The leading research institution formulating the overall study design is PUTH, which is 
Participating hospitals per province

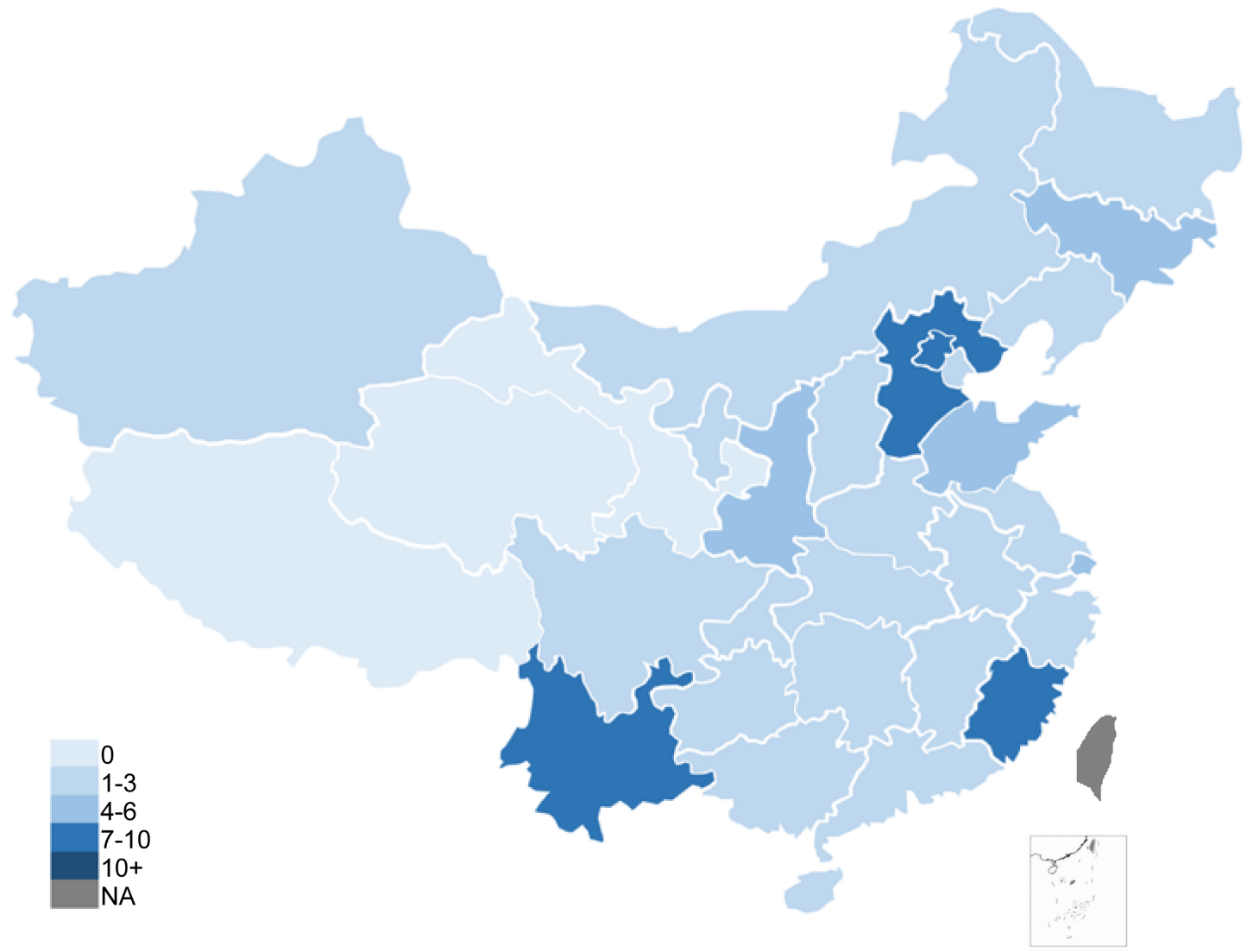

Province

Anhui

Beijing

Chongqing

Fujian

Gansu

Guangdong

Guangxi

Guizhou

Hainan

Hebei

Heilongjiang

Henan

Hubei

Hunan

Jiangsu

Jiangxi

Jilin

Liaoning

Neimenggu

Ningxia

Qinghai

Shaanxi

Shandong

Shanghai

Shanxi

Sichuan

Taiwan

Tianjin

Xizang

Xinjiang

Yunnan

Zhejiang

Numbers

3

3

Figure 2 Regions of mainland China with centres participating in CHALSR. NA, not available.

the largest ALS centre in China, with an ALS cohort of more than 2000 patients and approximately 500 newly diagnosed ALS patients per year. ${ }^{22}$

\section{Measures and data collection points}

The measurement and data collection time points of this study are shown in table 1 .

\section{Baseline measures}

The baseline data of interest will be obtained through face-to-face personal interviews by an experienced neurologist in the outpatient clinic at the time of recruitment to the study.

The measurements will include demographic information (eg, sex, age, ethnicity, education, occupation), lifestyle information (eg, food diary, mental status, physical activity, quality of life, environmental exposure), medical history (eg, individual medical history, family history, trauma history, reproductive history), diagnosis information (eg, date of diagnosis, age of diagnosis, level of diagnosis), clinical variables both at onset and during progression (eg, examination dates and sites, symptoms, physical examinations, electromyography tests, evaluations of disease severity) and treatment information (eg, use of medications, respiratory support, dietary assistance).

\section{Follow-up measures}

The following information of interest will be collected every 3 months through telephone calls or in-person interviews with patients or their caretakers by the trained investigators in the individual centres.

The measurements will include lifestyle information (eg, food diary, mental status, physical activity, quality of life, environmental exposure), clinical progression variables (eg, date and site of examination, symptoms, physical examinations, electromyography tests, evaluation of disease severity), treatment information (eg, use of medications, respiratory support, dietary assistance) and endpoint assessment.

\section{Outcome measures}

The primary outcome is effective survival. Tracheotomy and death due to any cause are defined as the endpoints of the study. In our study, effective survival is defined as the time between the onset of symptoms and our composite endpoints.

The secondary outcomes include overall survival, parameters related to disease progression and other descriptive results. In our study, overall survival is defined as the time between the onset of symptoms and death due to any cause. One of the typical measurements of disease progression is $\triangle \mathrm{FS}$, defined as the rate of change of the revised ALS Functional Rating Scale (ALSFRS-R). ${ }^{23}$ 
Table 1 Measurement and data collection time points

\begin{tabular}{|c|c|c|c|}
\hline Measures & Definitions & Baseline & Follow-up \\
\hline Demographic information & $\begin{array}{l}\text { Sex, age, date of birth, ethnicity } \\
\text { Dominant hand } \\
\text { Education, marriage, medical insurance, occupation }\end{array}$ & $\checkmark$ & \\
\hline Lifestyle information & $\begin{array}{l}\text { Food diary, mental status, physical activity, quality of life, } \\
\text { environmental exposure (toxicant) }\end{array}$ & $\checkmark$ & $\checkmark$ \\
\hline Medical history & $\begin{array}{l}\text { Individual medical history, family history, trauma history, } \\
\text { reproductive history }\end{array}$ & $\checkmark$ & \\
\hline Diagnosis information & $\begin{array}{l}\text { Date of diagnosis (fist and confirmed), delay of diagnosis, age of } \\
\text { diagnosis, level of diagnosis } \\
\text { Hospital of diagnosis, type of clinic (outpatient or inpatient) } \\
\text { Diagnosis of genetic test } \\
\text { Diagnosis of clinical phenotype }\end{array}$ & $\checkmark$ & \\
\hline $\begin{array}{l}\text { Variables of clinical } \\
\text { progression }\end{array}$ & $\begin{array}{l}\text { Date of progression, progression of affected sites } \\
\text { Progression of symptoms (motor symptoms/cognitive symptoms/ } \\
\text { nonmotor symptoms) } \\
\text { Progression of physical examination results } \\
\text { Progress of electromyography test results } \\
\text { ALS-FRS-R and } \triangle \text { ALS-FRS-R } \\
\text { Height, weight, BMI and } \triangle \text { height, weight, BMI } \\
\text { - Lung function (FVC, FVC\%) and } \Delta \text { lung function (FVC, FVC\%) }\end{array}$ & $\checkmark$ & $\checkmark$ \\
\hline Endpoint events & $\begin{array}{l}\text { Tracheotomy } \\
\text { Death due to any cause }\end{array}$ & & $\checkmark$ \\
\hline
\end{tabular}

ALS-FRS-R, amyotrophic lateral sclerosis functional rating scale-revised; BMI, body mass index; FVC, forced vital capacity; $\Delta=$ rate of decline from onset to confirmed diagnosis (for baseline) or from the last follow-up to this follow-up (for follow-up).

The ALSFRS-R is a questionnaire containing 12 questions designed to quantify the functional impairment of patients with ALS. This instrument evaluates respiratory function, speech and mobility of all four limbs. Each question has a maximum score of 4 , and a higher score on the scale represents a better functional status. Thus, the prediagnosis $\triangle \mathrm{FS}$ is determined as follows: (48 minus ALSFRS-R score of diagnosis)/(date of diagnosis minus date of onset). The postdiagnosis $\Delta \mathrm{FS}$ is determined as follows: (ALSFRS-R score of the last follow-up minus ALSFRS-R score of diagnosis)/(date of follow-up minus date of diagnosis). Similarly, other parameters such as $\triangle$ weight, $\triangle \mathrm{BMI}$ and $\triangle \mathrm{FVC}$ (forced vital capacity) before and after diagnosis will be included. Some descriptive results such as the cause of death, the timeline of progression at the affected sites and the duration from invasive ventilation to death will also be included as outcomes.

\section{Tools used for data collection (http://www.chalsr.net/)}

In this study, a purpose-designed internet-based platform will be used for data collection (figure 3). The online system guarantees a standard operating procedure for data management, including unified case report forms filled out by the responsible doctors, the supervision of 


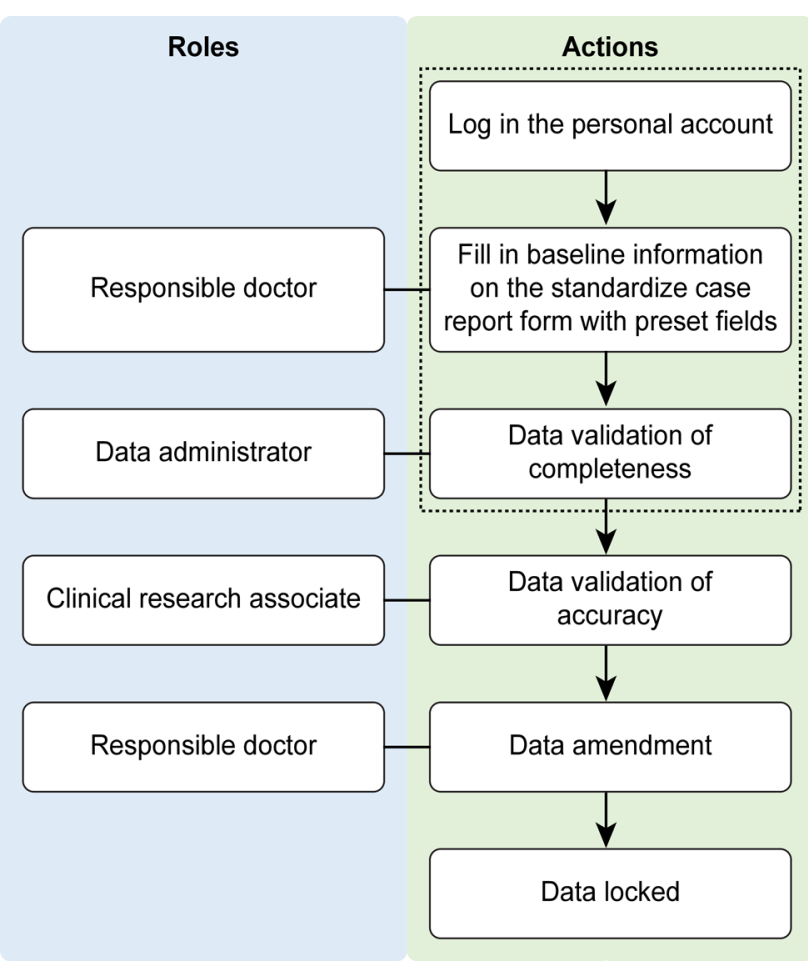

Figure 3 Management of data through the CHALSR internet-based system.

data completeness by data administrators and the supervision of data accuracy by clinical research associates. Each participating neurologist will have a personal account that allows him or her to upload the required information of patients. Password protection for the account will ensure the security of the data. Data validation will be conducted every 3 months by independent investigators. The data will be locked once they pass inspection or are amended by the responsible doctors according to the inspection. All the procedures can be completed through either the full website or a cell phone application to improve efficiency.

\section{Data analysis}

All analyses will be performed using SAS V.9.4, and a twosided $p<0.05$ will be considered statistically significant. We will report all the measures mentioned above. The continuous variables will be expressed as the mean, minimum and maximum or median, while the categorical variables will be expressed as proportions or frequencies. Patients will be divided into different subgroups according to their registry centres, disease phenotypes, treatments and other possible variations. Comparisons among subgroups will be conducted using parametric tests (one-way analysis of variance (ANOVA) or Student's t-test) or nonparametric tests ( $\chi^{2}$ test, Fisher's exact test, Kruskal-Wallis one-way ANOVA by ranks or Mann-Whitney U test). For the patients in general and each subgroup of patients, effective and overall survival curves will be estimated using Kaplan-Meier analysis, and important covariates will be compared using the log-rank test and Cox regression analysis.

\section{Patient and public involvement}

No patients or public were involved in the design, conduct, reporting or dissemination of this research study.

\section{ETHICS AND DISSEMINATION}

The study has been approved by the institutional ethics committee of PUTH (M2019388). Written informed consent will be obtained from each patient prior to enrolment, and all patient information will be treated as confidential. Our online system will assign each participant a serial number according to the time of registration. Only the responsible doctor could access the data. Individual centres could access their own data, and the principal investigator could access the integral data of all participating centres. The collective results of the study will be published in peer-reviewed journals and presented at relevant conferences.

\section{DISCUSSION}

Previous studies have defined ALS as a relentlessly progressive, fatal motor neuron disease with a variable natural history. ${ }^{24}$ The aetiology of ALS remains obscure. Environmental factors, including smoking, ${ }^{25}$ exposure to heavy metals ${ }^{26}$ and pesticides ${ }^{27}$ and more than 30 causative genes are associated with the pathogenesis of the disease. ${ }^{28}$ On the other hand, the prognosis of patients varies greatly, with survival ranging from several months to more than 10 years, which may be attributed to different phenotypes of ALS. ${ }^{16}$

The low prevalence and high heterogeneity of ALS severely limit the feasibility of the research. However, large disease registers could provide longitudinal and cross-sectional data. In ALS, large registers include clinical-based studies and population-based studies. Each kind of studies has its own advantages: ${ }^{29}$ clinical-based study could improve the case ascertainment; populationbased study could improve the generalisability since it is designed to detect all cases of a specific disease in a population defined by geographic boundaries. It could provide sufficient statistical power and avoid cohort bias. Previous population-based studies of ALS have reported similar prognostic factors. The result indicates that these factors are transportable between different cohorts. For example, EURALS in Europe, covering a total population of about 47.7 million person years, provided incidence rates of 2.6/100 000 person-years and prevalence rates of 7-9/100 000 persons. ${ }^{6}$ And EUROMOTOR ascertains a series of potential environmental risks for ALS including alcohol consumption, smoking, electric shock and other exposures. ${ }^{30-33}$ However, most of large registers are established in western countries and the diversity of ALS among different ethnicity calls for further cohorts. Although China has a large population, which implies a considerable number of patients with ALS, there is no large-scale registry study for ALS so far, which limits the representativeness of the previous research. Therefore, large cohort 
studies involving patients from different centres in mainland China with regular follow-ups are urgently needed to provide a more comprehensive profile of ALS.

To the best of our knowledge, this study is the first multicentre prospective cohort study in mainland China. We are taking full advantage of the large population of China, which guarantees that the scale of the study will be large as well. The multicentre participation and regular follow-ups will allow us to explore both the geographical and temporal characteristics of ALS. The study will be led by an experienced national ALS institution and supervised by the principal investigators from all participating hospitals, which will assure the professionalism of the overall study. Participating hospitals nationwide will benefit from this standard operating procedure for ALS and local clinical and scientific management will improve. In addition, the specially designed internet-based platform for the study will both unify the overall data and also improve efficiency and accuracy. This network-based information management system could lay the foundation for AI-enabled study and pave the way for translational work on ALS. We hope our effort to establish a standardised platform can be generalised from nationwide to worldwide application in the future. ${ }^{2021}$

Our study has several limitations. First, this study uses an internet-based platform, which requires all the participating doctors to have access to smart phones or computers. This may induce biases due to the lack of hardware equipment in rural areas. Second, as perception varies from one individual to another, some variation between centres in subjective evaluations such as physical examinations and ALS-FRS-R assessment cannot be avoided. Third, it would be difficult to obtain informed consent from all patients with ALS, and the centres involved in our study do not cover all provinces in mainland China. However, the study includes centres with a wide geographical distribution through China, and we will make a great effort to train the participating investigators in a standardised method to minimise the bias mentioned above.

In summary, our proposed multicentre cohort study with regular follow-ups will provide great insight into the clinical profile of ALS and fulfil the unmet need for a large-scale ALS study in mainland China. We hope to establish a comprehensive internet-based database for ALS through nationwide collaboration. Hence, our study could benefit both the clinical and scientific management of ALS, and we hope the pattern of this nationwide study in mainland China will serve as a good model for worldwide collaboration on ALS research.

Contributors All authors contributed to the conception and design of the study. HJ (Ji He), FJ and CL contributed to writing the protocol. HJ (Ji He) and FJ participated in revising and editing the draft manuscript. LN and $\mathrm{HJ}(\mathrm{Ji} \mathrm{He})$ and CL participated in the statistical analysis. HJ (Jing He), DJ, ZZ and MS collected important background information related to the study and acquisition of data. FD acted as the project's principal investigator, and contributed to study supervision. All authors read and approved the final manuscript before its publication.
Funding This project is funded by the National Natural Science Foundation of China (81974197, 81030019).

Map disclaimer The depiction of boundaries on this map does not imply the expression of any opinion whatsoever on the part of BMJ (or any member of its group) concerning the legal status of any country, territory, jurisdiction or area or of its authorities. This map is provided without any warranty of any kind, either express or implied.

Competing interests None declared.

Patient and public involvement Patients and/or the public were not involved in the design, or conduct, or reporting or dissemination plans of this research.

Patient consent for publication Not required.

Ethics approval The study was approved by the ethics committee of PUTH in March 2020.

Provenance and peer review Not commissioned; externally peer reviewed.

Open access This is an open access article distributed in accordance with the Creative Commons Attribution Non Commercial (CC BY-NC 4.0) license, which permits others to distribute, remix, adapt, build upon this work non-commercially, and license their derivative works on different terms, provided the original work is properly cited, appropriate credit is given, any changes made indicated, and the use is non-commercial. See: http://creativecommons.org/licenses/by-nc/4.0/.

ORCID iD

Dongsheng Fan http://orcid.org/0000-0002-6679-0864

\section{REFERENCES}

1 Tsai M-J, Hsu C-Y, Sheu C-C. Amyotrophic lateral sclerosis. N Engl J Med 2017;377:1602.

2 van Es MA, Hardiman O, Chio A, et al. Amyotrophic lateral sclerosis. Lancet 2017;390:2084-98.

3 Moore A, Young CA, Hughes DA. Economic studies in motor neurone disease: a systematic methodological review. Pharmacoeconomics 2017;35:397-413.

4 GBD 2016 Motor Neuron Disease Collaborators. Global, regional, and national burden of motor neuron diseases 1990-2016: a systematic analysis for the global burden of disease study 2016. Lancet Neurol 2018;17:1083-97.

$5 \mathrm{Xu} \mathrm{L}$, Liu T, Liu L, et al. Global variation in prevalence and incidence of amyotrophic lateral sclerosis: a systematic review and metaanalysis. J Neurol 2020;267:944-53.

6 Logroscino G, Traynor BJ, Hardiman O, et al. Incidence of amyotrophic lateral sclerosis in Europe. J Neurol Neurosurg Psychiatry 2010;81:385-90.

7 D'Ovidio F, Rooney JPK, Visser AE, et al. Critical issues in ALS case-control studies: the case of the Euro-MOTOR study. Amyotroph Lateral Scler Frontotemporal Degener 2017;18:411-8.

8 Beghi E, Millul A, Micheli A, et al. Incidence of ALS in Lombardy, Italy. Neurology 2007;68:141-5.

9 Rooney J, Vajda A, Heverin M, et al. Spatial cluster analysis of population amyotrophic lateral sclerosis risk in Ireland. Neurology 2015;84:1537-44.

10 Hardiman O, Al-Chalabi A, Brayne C, et al. The changing picture of amyotrophic lateral sclerosis: lessons from European registers. $J$ Neurol Neurosurg Psychiatry 2017;88:557-63.

11 Xu L, Chen L, Wang S, et al. Incidence and prevalence of amyotrophic lateral sclerosis in urban China: a national populationbased study. J Neurol Neurosurg Psychiatry 2020;91:520-5.

12 Chen L, Zhang B, Chen R, et al. Natural history and clinical features of sporadic amyotrophic lateral sclerosis in China. $J$ Neurol Neurosurg Psychiatry 2015;86:1075-81.

13 Marin B, Logroscino G, Boumédiene F, et al. Clinical and demographic factors and outcome of amyotrophic lateral sclerosis in relation to population ancestral origin. Eur J Epidemiol 2016;31:229-45.

14 Zaldivar T, Gutierrez J, Lara G, et al. Reduced frequency of ALS in an ethnically mixed population: a population-based mortality study. Neurology 2009;72:1640-5.

15 Roberts AL, Johnson NJ, Chen JT, et al. Race/ethnicity, socioeconomic status, and ALS mortality in the United States. Neurology 2016;87:2300-8.

16 Westeneng H-J, Debray TPA, Visser AE, et al. Prognosis for patients with amyotrophic lateral sclerosis: development and validation of a personalised prediction model. Lancet Neurol 2018;17:423-33. 
17 Nagel G, Unal H, Rosenbohm A, et al. Implementation of a population-based epidemiological rare disease registry: study protocol of the amyotrophic lateral sclerosis (ALS)--registry Swabia. BMC Neurol 2013;13:22.

18 Raymond J, Oskarsson B, Mehta P, et al. Clinical characteristics of a large cohort of US participants enrolled in the National amyotrophic lateral sclerosis (ALS) registry, 2010-2015. Amyotroph Lateral Scler Frontotemporal Degener 2019;20:413-20.

19 Zive DM, Cook J, Yang C, et al. Implementation of a novel electronic health Record-Embedded physician orders for life-sustaining treatment system. J Med Syst 2016;40:245.

20 Cui Y, Zhou X, Han J. China launched a pilot project to improve its rare disease healthcare levels. Orphanet J Rare Dis 2014;9:14.

21 Feng S, Liu S, Zhu C, et al. National rare diseases registry system of China and related cohort studies: vision and roadmap. Hum Gene Ther 2018;29:128-35.

22 Dorst J, Chen L, Rosenbohm A, et al. Prognostic factors in ALS: a comparison between Germany and China. J Neurol 2019;266:1516-25

23 Kimura F, Fujimura C, Ishida S, et al. Progression rate of ALSFRS-R at time of diagnosis predicts survival time in ALS. Neurology 2006;66:265-7.

24 Mejzini R, Flynn LL, Pitout IL, et al. Als genetics, mechanisms, and therapeutics: where are we now? Front Neurosci 2019;13:1310.

25 Armon C. Smoking may be considered an established risk factor for sporadic ALS. Neurology 2009;73:1693-8.
26 Yu Y, Su F-C, Callaghan BC, et al. Environmental risk factors and amyotrophic lateral sclerosis (ALS): a case-control study of ALS in Michigan. PLoS One 2014;9:e101186.

27 Malek AM, Barchowsky A, Bowser R, et al. Pesticide exposure as a risk factor for amyotrophic lateral sclerosis: a meta-analysis of epidemiological studies: pesticide exposure as a risk factor for ALS. Environ Res 2012;117:112-9.

28 Taylor JP, Brown RH, Cleveland DW. Decoding ALS: from genes to mechanism. Nature 2016;539:197-206.

29 Logroscino G, Traynor BJ, Hardiman O, et al. Descriptive epidemiology of amyotrophic lateral sclerosis: new evidence and unsolved issues. J Neurol Neurosurg Psychiatry 2008;79:6-11.

30 Peters S, Visser AE, D'Ovidio F, et al. Effect modification of the association between total cigarette smoking and ALS risk by intensity, duration and time-since-quitting: Euro-MOTOR. J Neurol Neurosurg Psychiatry 2020;91:33-9.

31 Peters S, Visser AE, D'Ovidio F, et al. Associations of electric shock and extremely low-frequency magnetic field exposure with the risk of amyotrophic lateral sclerosis. Am J Epidemiol 2019;188:796-805.

32 Rooney JPK, Visser AE, D'Ovidio F, et al. A case-control study of hormonal exposures as etiologic factors for ALS in women: EuroMOTOR. Neurology 2017;89:1283-90.

33 D'Ovidio F, Rooney JPK, Visser AE, et al. Association between alcohol exposure and the risk of amyotrophic lateral sclerosis in the Euro-MOTOR study. J Neurol Neurosurg Psychiatry 2019;90:11-19. 